\title{
Acidente vascular cerebral isquêmico em paciente portador de forame oval pérvio: estudo de caso
}

\author{
Ischemic stroke in a patient with patent foramen ovale: case study \\ Accidente cerebrovascular isquémico en paciente con foramen oval permeable: estudio de \\ caso
}

Bárbara Alvim Raposo Lemos ${ }^{1 *}$, Fauzer Cury Ochsendorf ${ }^{1}$, Maria Eduarda Calazans Resende ${ }^{1}$.

\begin{abstract}
RESUMO
Objetivo: Relatar o caso de paciente portador de Forame Oval Pérvio (FOP), que evoluiu com Acidente Vascular Cerebral (AVC) criptogênico, correlacionando com os dados disponíveis na literatura acerca da fisiopatologia, quadro clínico, diagnóstico e tratamento. Detalhamento de caso: Paciente F.C.L., sexo masculino, 48 anos, sem comorbidades, apresentou episódio de visão turva associado com desvio de rima à esquerda e perda de força em membro superior esquerdo, com retorno em menos de 24 horas. Realizou ressonância magnética de crânio que comprovou AVC isquêmico. A ecocardiografia transesofágica confirmou o diagnóstico de FOP. Vários exames complementares foram realizados, mas não houve associação com outra etiologia. A conduta estabelecida foi a oclusão percutânea transcateter do forame, juntamente com tratamento medicamentoso. Considerações finais: $E$ de suma importância o avanço nos estudos que englobam o AVC criptogênico e seu manejo, assim como sua associação com forame oval pérvio, já que o diagnóstico e tratamento interferem diretamente na sobrevida e na qualidade de vida do paciente.
\end{abstract}

Palavras-chave: Acidente vascular cerebral, Embolia paradoxal, Forame oval patente.

\begin{abstract}
Objective: To report the case of a patient with a Patent Foramen Ovale (PFO), who developed a cryptogenic stroke, correlating with the data available in the literature about pathophysiology, clinical picture, diagnosis and treatment. Case details: Male F.C.L., 48 years old, without comorbidities, presented an episode of blurred vision associated with left rhyme deviation and loss of strength in the left upper limb, with return in less than 24 hours. He underwent cranial magnetic resonance imaging which proved ischemic stroke. Transesophageal echocardiography confirmed the diagnosis of PFO. Several complementary tests were performed, but there was no association with another etiology. The established approach was percutaneous occlusion of the foramen, together with drug treatment. Final considerations: It is of utmost importance to advance in studies that include cryptogenic stroke and its management, as well as its association with a patent foramen ovale, since diagnosis and treatment directly interfere in the patient's survival and quality of life.
\end{abstract}

Key words: Stroke, Embolism paradoxical, Patent foramen ovale.

\section{RESUMEN}

Objetivo: Informar el caso de un paciente con Foramen Oval Permeable (FOP), que desarrolló un ictus criptogénico, correlacionando con los datos disponibles en la literatura sobre fisiopatología, cuadro clínico, diagnóstico y tratamiento. Detalle del caso: Hombre F.C.L., 48 años, sin comorbilidades, presentó un episodio de visión borrosa asociado a desviación de rima izquierda y pérdida de fuerza en miembro superior izquierdo, con retorno en menos de 24 horas. Se sometió a una resonancia magnética craneal que demostró accidente cerebrovascular isquémico. La ecocardiografía transesofágica confirmó el diagnóstico de FOP. Se realizaron varias pruebas complementarias, pero no hubo asociación con otra etiología. El abordaje establecido fue la oclusión percutánea del foramen, junto con el tratamiento farmacológico. Consideraciones finales: Es de suma importancia avanzar en estudios que incluyan el ictus criptogénico y su manejo, así como su asociación con un foramen oval permeable, ya que el diagnóstico y el tratamiento interfieren directamente en la supervivencia y calidad de vida del paciente.

Palabras clave: Accidente cerebrovascular, Embolia paradójica, Foramen oval permeable.

${ }^{1}$ Universidade de Franca (UNIFRAN), Franca - SP. *E-mail: babiraposo57@gmail.com 


\section{INTRODUÇÃO}

O Acidente Vascular Cerebral (AVC) configura-se atualmente como uma das principais causas de morbimortalidade no Brasil, sendo o tipo isquêmico responsável por aproximadamente $80 \%$ dos episódios. Este, causado pela oclusão da circulação cerebral, apresenta sua origem trombogênica desconhecida em até $40 \%$ dos casos, quando presente em pacientes jovens, e é, então, denominados de AVC criptogênico, e, em grande maioria, manifesta-se em pacientes atípicos, ou seja, com uma faixa etária de até 40 anos, sem a associação de fatores de risco comuns para o desenvolvimento de uma isquemia cerebral, como hipertensão arterial sistêmica (HAS), dislipidemia, tabagismo e Diabetes Mellitus (DM) (GRORY BM, et al., 2019; NEGRÃO EM, et al., 2012).

Dessa maneira, por comprometer indivíduos jovens e impactar negativamente nos índices socioeconômicos, estudos são desenvolvidos a fim de se estabelecer os fatores de risco obscuros ao surgimento do AVC criptogênico. Em 1877, por exemplo, durante um procedimento de necrópsia de uma mulher de 25 anos vítima de AVC, Cohnhein, patologista alemão, identificou a concomitância da manifestação isquêmica com a presença de um shunt cardíaco proveniente da patência do forame oval, estabelecendo, pela primeira vez, uma possível correlação entre os dois fatores (FUKUJIMA MM, et al., 2004; MAGALHÃES E, et al., 2006).

O forame oval é situado entre as câmaras cardíacas atriais e tem sua origem no período embrionário, cujo papel é facilitar, através do shunt cardíaco, a passagem direta do sangue placentário oxigenado para a circulação sistêmica fetal. Após o nascimento, a partir da instauração da circulação pulmonar, a câmara cardíaca esquerda sobrepõe significativamente a pressão presente na câmara direita, fechando, por mecanismos pressóricos, o canal de comunicação. Todavia, a não oclusão fisiológica do forame oval se faz presente em até $25 \%$ da população mundial, e em pacientes que apresentam AVC criptogênico esse índice alcança $45 \%$ dos indivíduos. A persistência desse canal após o nascimento denomina-se Forame Oval Patente ou Pérvio (FOP) (MARQUES M, et al., 2021; SAMPAIO AVS e UTAGAWA CY, 2014).

A relação causa-consequência de FOP e AVC criptogênico, contudo, não é bem estabelecida. O FOP é um fator predisponente ao desenvolvimento de embolia paradoxal, na qual os êmbolos provenientes da circulação tem sua comunicação facilitada pelo orifício interatrial, sem passar por uma rede capilar. Pesquisas recentes apontam que os trombos também podem ter origem no forame devido á estase sanguínea estabelecida e ativação dos mecanismos de coagulação. Os trombos alcançam então a circulação cerebral que, pelo menor calibre, é ocluída levando ao AVC (FURLAN AJ e JAUSS M, 2013; MOJADIDI MK, et al., 2018; SAMPAIO AVS e UTAGAWA CY, 2014; SPENCE JD, 2018).

A maioria dos pacientes, entretanto, não apresenta sinais ou sintomas sugestivos da permanência do forame ou que sustentem o pedido de exames detalhados. Dessa maneira, o diagnóstico, por vezes, é estabelecido após o surgimento de complicações secundárias ao FOP, como AVC, migrânea, insuficiência renal, síndrome descompressiva em mergulhadores, embolia periférica incluindo infarto do miocárdio e mal de Alzheimer (SAMPAIO AVS e UTAGAWA CY, 2014; VALDIVIESO CG, 2019).

Todos os pacientes que sofrem AVE devem realizar exames de imagem que mostrem as artérias cerebrais e cervicais, Ecocardiograma Transtorácico (ETT), pesquisa de hipertensão arterial sistêmica, hiperlipidemia, diabetes mellitus e tabagismo. Caso nenhum achado seja encontrado, exames complementares devem ser feitos e selecionados de forma individualizada, com intuito de elucidar a etiologia da doença. Quando há suspeita clínica de FOP, a investigação deve ser iniciada com o ETT com estudo de contraste salino. Para melhorar a sensibilidade, pode ser feito em repouso, com manobra de Valsalva e com tosse (MOJADIDI MK, et al., 2018; SAMPAIO AVS e UTAGAWA CY, 2014; SPENCE JD, 2018).

Na suspeita de shunt dinâmico, o ETT pode ser feito enquanto o paciente se exercita. Caso haja dúvida em relação aos achados, um Ecocardiograma Transesofágico (ETE) deve ser realizado, pois permite melhor análise do átrio, aurícula esquerda, septo interatrial, função ventricular, morfologia e funcionamento valvular. O Doppler Transcraniano (TCD) é outra modalidade de imagem que pode ser útil na detecção de shunt da direita para a esquerda e sinais microembólicos, podendo ser executado em repouso, exercício e com 
manobras provocativas (MOJADIDI MK, et al., 2018; SAMPAIO AVS e UTAGAWA CY, 2014; SPENCE JD, 2018).

Pacientes com FOP encontrado acidentalmente, assintomáticos, sem história de AVC ou sequelas pelo mesmo, e com exames de imagem sem alterações, não possuem indicação formal de tratamento. A terapia para prevenção secundária de AVC pode incluir medicamentos antiplaquetários, anticoagulantes orais, ou ainda o fechamento transcateter ou cirurgia aberta. Estudos mais recentes demonstram que o fechamento do FOP é mais eficaz na redução da recorrência de isquemias cerebrais do que a terapia medicamentosa, principalmente quando se trata de pacientes jovens e com grandes shunts (MOJADIDI MK, et al., 2018; SPENCE JD, 2018).

Dessa forma, objetivou-se relatar o caso de paciente portador de FOP, que evoluiu com AVC criptogênico, comprovado pela história clínica e exames laboratoriais e de imagem, correlacionando com os dados disponíveis na literatura acerca da fisiopatologia, quadro clínico, diagnóstico e tratamento.

\section{DETALHAMENTO DO CASO}

O presente artigo foi conduzido como estudo observacional do tipo estudo de caso. Termo de Consentimento Livre e Esclarecido (TCLE) foi elaborado de forma a elucidar o objetivo do estudo, a importância da participação do paciente, e a manutenção do anonimato a partir do aceite de colaboração pelo mesmo. Os riscos, questões éticas e dúvidas foram esclarecidos. O documento foi formulado em duas cópias, lido e assinado pelo participante e pelo pesquisador.

Paciente F.C.L, masculino, 48 anos, casado, programador de sistemas, branco, natural e residente de Uberaba-MG. Apresenta episódio de visão turva associado com desvio de rima à esquerda e perda de força em membro superior esquerdo, com retorno em menos de 30 minutos. Nega antecedentes familiares cardiovasculares ou cerebrovasculares, tabagismo e uso de medicações contínuas. Pratica atividade física regularmente. Havia realizado teste ergométrico e Ecocardiograma Transtorácico (ETT) há nove meses, com NYHA I, aptidão cardiorrespiratória regular, prolapso de valva mitral discreto, sem outras alterações.

Procura pronto-socorro (PS), uma hora após o início dos sintomas, onde foi realizada aferição de pressão arterial, exame neurológico sumário com testes de força e sensibilidade, ausculta cardíaca e pulmonar, sem alterações. Formulada hipótese diagnóstica de acidente isquêmico transitório (AIT), medicado e liberado.

Após três dias, apresenta quadro de perda de sensibilidade de membro inferior esquerdo e cefaleia intermitente. Solicitada Ressonância Nuclear Magnética (RNM) encefálica, na qual apontou isquemia subaguda de artéria cerebral média direita, com acometimento dos giros pré-central e frontal médio.

A partir dos dados clínicos e resultado da RNM encefálica, foi internado para maior investigação. Realizado doppler das carótidas e vertebrais, ultrassonografia de ombro, tomografia computadorizada de tórax, teste rápido para covid-19, hemograma, glicemia e ETT, sem alterações. Recebeu alta com bissulfato de clopidogrel $75 \mathrm{mg}$ e sinvastatina $20 \mathrm{mg}$.

Procurou serviço de cardiologia, onde foi realizada angiorressonância arterial do crânio, angiotomografia arterial do pescoço, hemograma completo, ferritina, capacidade de fixação do ferro, glicemia, hemoglobina glicada, lipidograma, hemocisteína, função renal e hepática, eletrólitos, eletroforese de proteínas, anti-HIV 1 e 2, anticorpos anti-treponema pallidum, TSH, T4 livre, vitamina B12, vitamina D3, ácido fólico, CPK, PCR e VHS $1^{\underline{a}}$ hora, sem alterações.

O Ecocardiograma transesofágico mostrou insuficiência mitral discreta, forame oval pérvio, tunelizado, com extensão aproximada de nove milímetros, e altura máxima do descolamento de três milímetros, com discreta mobilidade; ausência de trombo intracavitário e desempenho sistólico preservado (Figura 1). 
Figura 1- Ecocardiograma transesofágico mostrando forame oval pérvio de extensão aproximada de $9 \mathrm{~mm}$ e altura máxima do descolamento de 3 $\mathrm{mm}$, com discreta mobilidade.

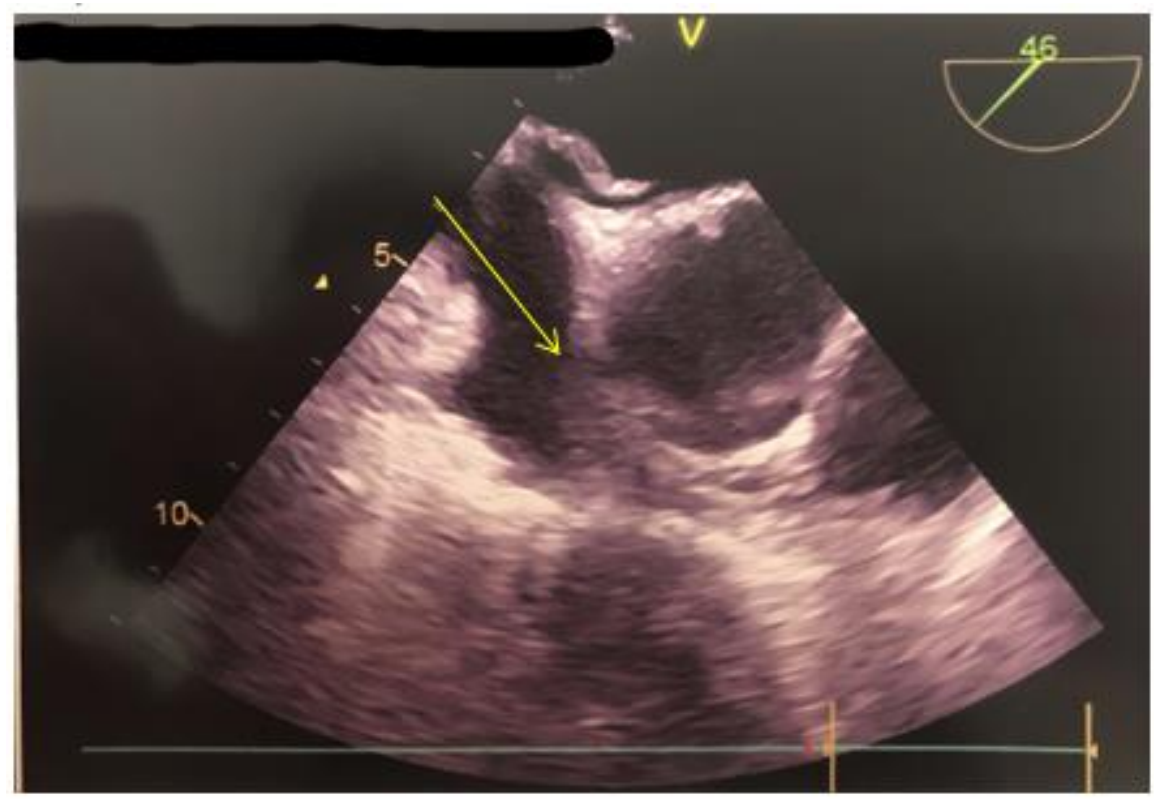

Fonte: Lemos B, et al., 2021.

Foi submetido a cateterismo cardíaco acompanhado de ETE para inserção de oclusor de FOP, e recebeu alta com ácido acetilsalicílico $100 \mathrm{mg}$ contínuo, bissulfato de clopidogrel $75 \mathrm{mg}$ e sinvastatina $20 \mathrm{mg}$. Obtevese melhora completa dos sintomas uma semana após o tratamento estabelecido (Figura 2).

Figura 2 - Prótese Cera 18/25 para Forame Oval Patente.

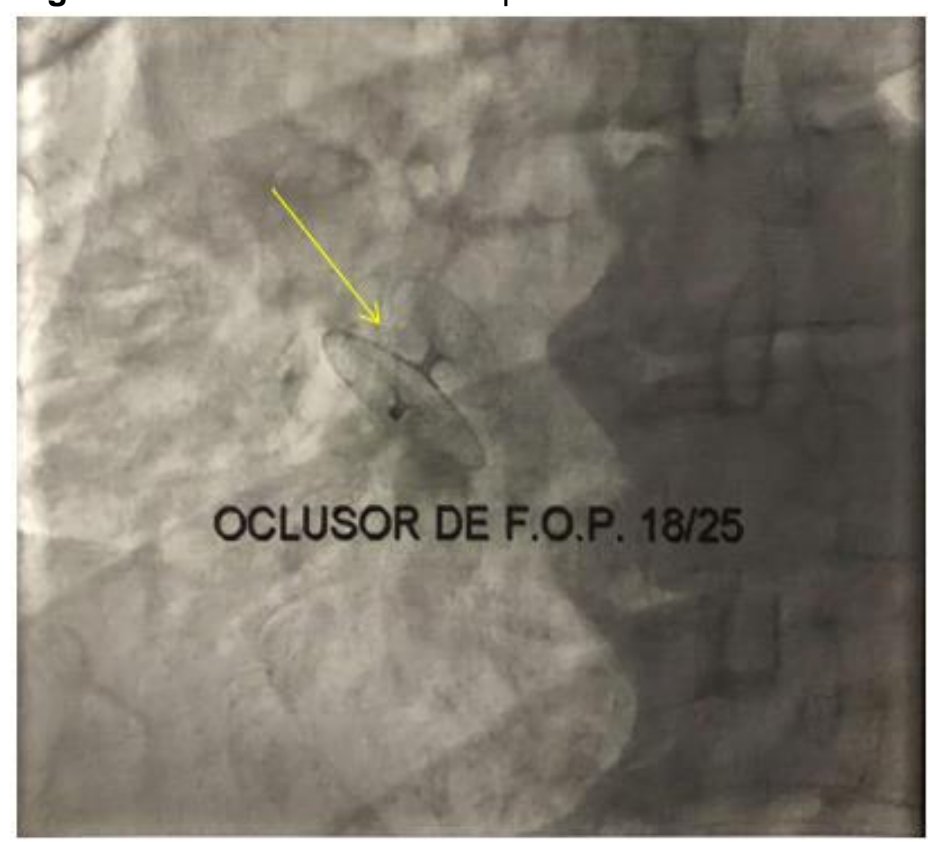

Fonte: Lemos B, et al., 2021.

\section{DISCUSSÃO}

O AVC isquêmico causado por FOP não possui mecanismo totalmente esclarecido. Entretanto, é bem documentado que a permanência de um canal entre os átrios permite shunt interatrial transitório, com o 
potencial de transmitir trombos paradoxais e sangue desoxigenado. Outros mecanismos estudados são a fibrilação atrial paroxística e a formação de trombos dentro do próprio forame, que contém sangue estagnado. Todavia, a demonstração histológica da semelhança dos trombos em acidentes criptogênicos, ou seja, sem causa definida, e cardioembólicos, reforça o mecanismo embólico do mesmo (MOJADIDI MK, et al., 2018; SAMPAIO AVS e UTAGAWA CY, 2014; SPENCE JD, 2018).

O FOP está presente em cerca de $25 \%$ da população, e a maioria dos casos é diagnosticada de forma acidental durante a investigação de outras patologias, principalmente cardíacas, pois o defeito anatômico em si não se associa a quadro clínico específico. Entretanto, sinais e sintomas podem surgir a partir de síndrome descompressiva, migrânea, infarto do miocárdio, insuficiência renal ou acidentes vasculares cerebrais isquêmicos, como ocorreu no caso estudado. Sendo assim, a sintomatologia aparece associada ao território arterial acometido, que no estudo em questão foi a artéria cerebral média direita, com consequente isquemia nos giros pré-central e frontal médio (MOJADIDI MK, et al., 2018; SAMPAIO AVS e UTAGAWA CY, 2014; SPENCE JD, 2018).

Esse evento aparece, na maioria dos casos, como AVE criptogênico em pacientes com menos de 45 anos e sem fatores de risco conhecidos, sendo a incidência do mesmo decrescente com o aumento da idade e com a presença de tais fatores. Em contrapartida, no caso estudado os sintomas iniciais duraram menos de um minuto, e o exame físico estava normal, e por isso, foi levantada a hipótese de acidente isquêmico transitório (AIT). Porém, a partir dos guidelines da AHA/ASA, AIT passou a ser caracterizado como evento agudo de isquemia encefálica, sem infarto cerebral, o que não ocorreu neste estudo (SAMPAIO AVS e UTAGAWA CY, 2014).

O paciente do caso apresentava idade atípica para FOP, com 48 anos no momento do evento, todavia, a hipótese foi estabelecida após realização de muitos exames complementares, todos negativos para as causas mais comuns de AVE, pela confirmação da presença do forame oval patente através do ecocardiograma transesofágico, e pela ausência de fatores de risco para outra etiologia.

O diagnóstico de FOP é feito a partir da detecção por exames de imagem da presença de shunt interatrial ou identificação da permanência do canal. São elegíveis atualmente três exames, o Doppler transcraniano (TCD) o ecocardiograma transtorácico (ETT) e o ecocardiograma transesofágico (ETE), variando quanto aos índices de sensibilidade e especificidade. O TCD identifica o FOP através da presença de microêmbolos na circulação, contudo, a depender do grau de abertura do forame, mesmo com a presença de FOP, não necessariamente haverá a identificação de shunt, o que reduz sua sensibilidade (DATTILO PB, et al., 2013; DUARTE LF, et al., 2019; VALDIVIESO CG, 2019).

A todos os pacientes que apresentam quadro de isquemia cerebral de origem desconhecida, é indicada uma triagem diagnóstica com ETT, exame de fácil execução, não invasivo, capaz de avaliar anormalidades em câmaras cardíacas como a presença do forame oval patente. O ETT, por outro lado, embora apresente uma boa especificidade possui índices de falso negativos consideráveis devido a uma baixa sensibilidade para positivar o FOP quando este apresenta um canal de abertura relativamente pequeno e shunt com baixo volume (DATTILO PB, et al., 2013; DUARTE LF, et al., 2019; VALDIVIESO CG, 2019).

Dessa maneira, o ETE é padrão-ouro para o diagnóstico atualmente, e apesar da desvantagem de ser um exame mais invasivo, desconfortável ao paciente e necessitar de sedação, apresenta uma melhor acurácia diagnóstica para canais interatriais menores, além de permitir uma avaliação mais ampla e fidedigna da anatomia e morfologia cardíaca, bem como da fisiologia valvar. No caso estudado, somente este exame conseguiu estabelecer o diagnóstico de FOP e fazer uma possível associação com o acidente vascular cerebral sofrido pelo paciente, já que todos os demais exames não apresentaram alterações (DATTILO PB, et al., 2013; DUARTE LF, et al., 2019; VALDIVIESO CG, 2019).

A escolha terapêutica para pacientes com FOP é variável e individualizada, respeitando o quadro clinico apresentado, assim como as comorbidades associadas. A literatura demonstra que para pacientes que não apresentam comorbidades secundárias ao FOP ou quadros isquêmicos, é desnecessário tratamentos específicos, conquanto, para aqueles que apresentarem quadro de isquemia cerebral, como o paciente 
relatado, ou outras comorbidades secundárias, os índices de recidivas alcançam 11\%, logo, há um riscobenefício positivo ao paciente ao se estabelecer um plano terapêutico, seja por tratamento clínico, seja pelo fechamento do canal (DUARTE LF, et al., 2019; TATANI SB, et al., 2001).

A conduta clínica possui o objetivo de evitar a formação trombogênica com a administração regular de antiplaquetários e anticoagulantes. As indicações quanto ao fechamento cirúrgico do canal são defendidas pelo fato de eliminarem o fator de risco para um segundo AVC, com consequente queda das recidivas. Tal conduta é viabilizada por dois métodos, por via aberta, com maior risco de mortalidade e complicações póscirúrgicas, ou por via percutânea transcateter, uma opção bem aceita atualmente por ser minimamente invasiva, realizada através de cateterismo e com período de internação reduzido (AGASTHI P, et al., 2019; DATTILO PB, et al., 2013; DUARTE LF, et al., 2019; SAVER JL, et al., 2017).

Os pacientes que se beneficiam por tais técnicas devem, contudo, receber alta hospitalar com uma terapia anticoagulante associada a fim de reduzir os mecanismos fisiológicos de coagulação, inibindo a formação trombogênica e as consequências embólicas. Para o paciente relatado, apesar de episódio isquêmico único, foi optado pela realização de prevenção secundária por cateterismo percutâneo, pelo bom prognóstico, risco de reincidência de $\mathrm{AVCl}$ criptogênico, por ser jovem e não possuir comorbidades que contraindicassem a escolha cirúrgica, associado a medidas farmacológicas posteriores (DATTILO PB, et al., 2013).

O paciente deste estudo é jovem e sem fatores de risco típicos para AVC. Todavia, foi realizada investigação completa, descartando etiologias mais prováveis, e posteriormente buscando etiologias menos comuns. Somente FOP foi identificado. A partir disso, optou-se pelo fechamento percutâneo do forame e terapia medicamentosa. Sendo assim, é de suma importância o avanço nos estudos que englobam o AVC criptogênico e seu manejo, assim como sua associação com FOP, já que o diagnóstico e tratamento interferem diretamente na sobrevida e na qualidade de vida do paciente.

\section{REFERÊNCIAS}

1. AGASTHI P, et al. Are we there yet with patent foramen ovale closure for secondary prevention in cryptogenic stroke? A systematic review and meta-analysis of randomized trials. SAGE Open Medicine, 2019; 7: 2050312119828226.

2. DATTILO PB, et al. Patent foramen ovale. Cardiology Clinics, 2013; 31(3): 401-415.

3. DUARTE LF, et al. Acidente vascular cerebral isquêmico em paciente portador de forame oval patente: relato de caso. Revista de Saúde, 2019; 10(2): 43 - 48.

4. FUKUJIMA MM, et al. Forame oval patente e acidente vascular cerebral. Revista Neurociências, 2004; 12(4): 209211.

5. FURLAN AJ e JAUSS M. Patente foramen ovale and cryptogenic stroke: The hole story. Aha Journal, 2013; 44(9): 2676-2678.

6. GRORY BM, et al. Cryptogenic stroke: Diagnostic Workup and Management. Curr Treat Options Cardiovasc Med, 2019; 21(11).

7. MAGALHÃES E, et al. Papel do forame oval patente e da válvula de Eustáquio nos eventos tromboembólicos. Arquivos de Neuro-Psiquiatria, scielo, 2006; 64: 245-248.

8. MARQUES M, et al. Forame oval patente - revisão de literatura. Patent oval foramen - literature review. Brazilian Journal of Health Review, 2021; 4(1): 2578-2585.

9. MOJADIDI MK, et al. Cryptogenic Stroke and Patent Foramen Ovale. J Am Coll Cardiol., 2018; 71(9): $1035-1043$.

10. NEGRÃO EM, et al. Forame oval patente e acidente vascular cerebral isquêmico em pacientes jovens: Associação causal ou estatística?. Sociedade Brasileira de Cardiologia, 2012; 34(12): 128-139.

11. SAMPAIO AVS e UTAGAWA CY. Forame oval patente e acidente vascular cerebral: causa e consequência? Patent foramen ovale and stroke: cause and consequence?. Cadernos UniFOA, 2014; 26: 131-136.

12. SAVER JL, et al. Long-Term Outcomes of patent foramen ovale closure or medical therapy after stroke. The New England Journal of Medicine, 2017; 377(11): 1022-1032.

13. SPENCE JD. Cardioembolic stroke: Everything has changed. Revista Stroke and Vascular Neurology, 2018; 3(2): 76-83.

14. TATANI SB, et al. Impacto clínico da ecocardiografia transesofágica em pacientes com acidente vascular cerebral sem evidência clínica de fonte emboligênica cardíaca. Arq. Brasileiro de Cardiologia, 2001; 76(6): 453-457.

15. VALDIVIESO CG. Foramen oval permeable e ictus criptogénico. Revista Uruguaia de Cardiologia, 2019; 34(2): 185191. 